\title{
Multilinguales
}

\section{L'enseignement/apprentissage de la grammaire dans le collège algérien : quelle place pour la démarche inductive?}

The teaching / learning of grammar in the algerian middle school: what place for the inductive approach?

$$
\text { لدنهريس / تعلم قواعد اللغة في المدارس المتوسطة الجزائرية:أي مكان }
$$

\section{Zouina Hocini}

\section{OpenEdition}

\section{Journals}

Édition électronique

URL : http://journals.openedition.org/multilinguales/1417

DOI : $10.4000 /$ multilinguales. 1417

ISSN : 2335-1853

Éditeur

Université Abderrahmane Mira - Bejaia

\section{Référence électronique}

Zouina Hocini, «L'enseignement/apprentissage de la grammaire dans le collège algérien : quelle place pour la démarche inductive? », Multilinguales [En ligne], 9 | 2018, mis en ligne le 01 juin 2018, consulté le 21 décembre 2020. URL : http://journals.openedition.org/multilinguales/1417 ; DOI : https://doi.org/ 10.4000/multilinguales. 1417

Ce document a été généré automatiquement le 21 décembre 2020.

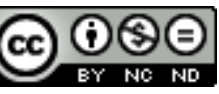

Multilinguales est mise à disposition selon les termes de la Licence Creative Commons Attribution Pas d'Utilisation Commerciale - Pas de Modification 4.0 International 


\title{
L'enseignement/apprentissage de la grammaire dans le collège algérien : quelle place pour la démarche inductive?
}

\author{
The teaching / learning of grammar in the algerian middle school: what place \\ for the inductive approach? \\ تدريس / تعلم قواعد اللغة في المدارس المتوسطة الجزائرية: أي مكان \\ للنهج الاستقرائي؟ ت لتعلم
}

\section{Zouina Hocini}

1 D'aucuns s'accordent à dire que l'on ne peut faire abstraction de la grammaire et de son apprentissage en classe de FLE. Celle-ci est, en effet, au cœur de la pratique enseignante. Selon Besse et Porquier : «(...) la grammaire est, très précisément, ce qu'on ne peut éviter dès qu'on fait un usage approprié d'une langue» (1991: 72). Ils rajoutent un peu plus loin: "c'est pourquoi la didactique des langues ne peut contourner le problème de l'enseignement/apprentissage des régularités en particulier morphosyntaxiques » (Idem).

Dans la même optique, Fougerouse estime, en parlant de la grammaire, que :

Cette composante linguistique, qui a connu une période de disgrâce, une sorte de passage à vide dans les années soixante et soixante-dix, semble revenue en force aujourd'hui dans la classe de langue. Ce retour démontre qu'elle est incontournable pour quiconque veut apprendre à communiquer en français. (2001:165).

Si l'enseignement de la grammaire, en classe de FLE notamment, a fait l'objet de moult débats chez les didacticiens, il s'avère que sa démarche d'enseignement ne l'est pas moins. Ainsi, les uns préconisent une démarche d'enseignement implicite, tandis que les autres prônent une démarche explicite qu'elle soit déductive ou inductive :

En effet, un enseignement grammatical ne se résume pas aujourd'hui à une simple présentation des règles grammaticales à l'apprenant. Certains défendent un enseignement implicite et bannissent toute forme de métalangage; d'autres favorisent un mode explicite ayant recours à la déduction où les règles sont 
présentées à l'apprenant dès le départ; d'autres préconisent un mode explicite mais avec une démarche inductive en visant à faire découvrir les règles par l'apprenant (Martineau, 2007 : 13-14).

4 A la question de savoir comment améliorer l'enseignement grammatical et de surcroit développer les compétences des élèves, Christian Dumarsais répond en ces termes :

Une solution possible est d'adopter une approche réflexive de la grammaire. Il s'agit de susciter un esprit de recherche et d'interrogation, en ce qui concerne le langage, en plaçant l'élève au premier plan de ses apprentissages de sorte qu'il puisse construire son savoir. Pour ce faire, il est possible de faire en classe trois activités de réflexion sur la langue : les ateliers de négociation graphique, la phrase dictée du jour et la démarche active de découverte. (2010 : 73-74).

5 Ce chercheur prône ainsi une activité réflexive de l'apprenant, ce que les didacticiens désignent sous l'appellation de « conceptualisation grammaticale ». Parmi ces activités, nous retrouvons la démarche active de découverte; une démarche inductive prônée essentiellement par Susanne-G Chartrand. Dès lors, en quoi consiste cette démarche? En quoi est-elle plus efficace en classe de FLE ? Dans quelle mesure les enseignants y recourent-ils?

\section{Description du corpus et méthodologie de la recherche}

6 Notre corpus se compose d'un ensemble de documents officiels utilisés pour enseigner le français dans l'enseignement moyen et de séances d'observation de classe.

7 Les documents officiels comprennent les manuels scolaires et les documents d'accompagnement des programmes des trois niveaux, à savoir la $2^{\text {ème }}, 3^{\text {ème }}$ et $4^{\text {ème }}$ année moyenne.

8 Pour recueillir des informations et compléter notre analyse, nous avons opté pour l'observation des classes. Notre corpus est extrait de séquences filmées, transcrites, allant de 48 à 55 minutes. Nous avons pu réunir 12 séances filmées (3 séances avec la 2 ème $\mathrm{AM}, 5$ avec la $3^{\text {ème }} \mathrm{AM}$ et 4 avec la $4^{\text {ème }} \mathrm{AM}$ ) dans deux collèges différents de la ville de Bejaia. Celles-ci ont été stockées dans des cédéroms. Nous nous sommes basée sur une transcription orthographique en nous référant à la convention de transcription de linguistes, comme Evelyne Pochon-Berger. (2011: 144)

9 Après négociation avec les trois enseignantes concernées, nous avons convenu d'entamer les séances d'observation dès le début du cours sur la relative. Après une autre négociation avec les concernées, nous avons pu assister à d'autres séances, qui ne sont certainement pas conséquentes mais que nous estimons satisfaisantes pour notre recherche. En outre, le cours sur notre fait de langue se fait au troisième trimestre, période assez courte n'étant pas tout à fait propice à l'enquête. Ainsi nous avons convenu d'assister aux séances une à deux fois par semaine selon les emplois du temps des enseignants. 


\section{Cadre situationnel de l'enseignement du FLE au collège algérien}

D'après la loi d'orientation sur l'éducation nationale, l'enseignement du FLE est tributaire du développement intellectuel et moral de l'apprenant lui assurant ainsi un avenir non seulement professionnel mais aussi social :

L'école algérienne a pour vocation de former un citoyen doté de repères nationaux incontestables, profondément attaché aux valeurs du peuple algérien, capable de comprendre le monde qui l'entoure, de s'y adapter et d'agir sur lui et en mesure de s'ouvrir sur la civilisation universelle (MEN, 2012/2013b : 5).

Par ailleurs, cet intérêt pour le français se manifeste notamment à travers le volume horaire qui lui est octroyé. Ainsi, au cycle moyen, cette matière bénéficie de 5 heures par semaine ( 4 heures de cours et 1 heure de travaux dirigés). Cela dit, malgré les efforts consentis par le corps éducatif algérien, les difficultés d'acquisition de cette langue sont considérables et se traduisent essentiellement par des dysfonctionnements discursifs et grammaticaux. Ce qui nous pousse à nous intéresser particulièrement aux causes de ces difficultés.

Depuis la réforme de 2003, l'école algérienne est en constante évolution, suivant l'introduction de nouvelles méthodologies d'enseignement qui correspondent au changement de statuts de l'apprenant et de l'enseignant. En effet, ce dernier n'est plus le "maître ", " détenteur de savoir " mais plutôt un guide, un encadreur orientant l'apprenant. L'enseignement est de ce fait relégué au second plan au profit de l'apprentissage. L'apprenant est plus actif ; il construit lui-même son savoir, tel est le postulat de base des nouvelles approches adoptées dans le secteur éducatif algérien, à savoir l'approche communicative et l'approche par compétences :

L'approche par les compétences traduit le souci de privilégier une logique d'apprentissage centrée sur l'élève, sur ses actions et réactions face à des situations-problèmes, par rapport à une logique d'enseignement basée sur les savoirs et sur les connaissances à faire acquérir. (MEN, 2013f : 8)

13 Pour satisfaire aux exigences d'une nouvelle approche d'enseignement/apprentissage, les documents pédagogiques ne cessent de changer. Dans cet article, outre les séances d'observation, nous décrirons, d'emblée, puis analyserons ces documents pédagogiques, à savoir: les programmes, les documents d'accompagnement des programmes et les manuels scolaires.

Les points de langue sont abordés en grammaire suivant le projet ou la séquence didactique à réaliser. C'est ainsi que l'on retrouve la relative dans le second palier étant donné qu'elle sert la description dans les textes qui racontent un événement fictif ou réel (récit, fait divers, etc.). Mais, est-ce toujours le cas au sein des classes de FLE? Les enseignants suivent-ils cette démarche en situation de classe? Comment la grammaire est-elle enseignée?

\section{L'enseignement de la grammaire en classe de FLE}

15 Les enseignants recourent souvent à une démarche particulière dans l'enseignement/ apprentissage d'un point de langue. Nous nous intéressons, dans cette partie, au type de démarche d'enseignement de la grammaire. 


\section{Démarche d'enseignement grammatical}

Il existe plusieurs démarches d'enseignement, nous en aborderons ci-dessous trois, à savoir : le type implicite, explicite et inductif.

\section{Grammaire implicite}

La grammaire implicite correspond à un enseignement tacite en faisant abstraction de la règle et du métalangage qui lui est afférent. C'est à travers les exercices structuraux que nous aborderons ultérieurement- que ce type se manifeste. On parle ainsi de « silence métalinguistique » (Damar, 2009:61).

Toutefois, ce type de pratique semble présenter un certain nombre d'inconvénients. En effet, cela conduirait à l'apparition d'erreurs chez l'apprenant dans la mesure où les règles qu'il a acquises ne sont point contrôlées par l'enseignant : " On s'est rendu compte que, vu que l'enseignant ne cautionne pas la validité ni les limites de la règle acquise, ce type de démarche engendrait des erreurs de généralisation de la part des apprenants par analogie" (idem).

19 Nous postulons donc, à la suite des chercheurs (Besse et Porquier, Vigner), que la grammaire implicite n'est pas suffisante. C'est pourquoi elle doit être complétée par un enseignement explicite. Un principe inhérent aux approches communicatives qui : « (...) n'a pas rompu avec la grammaire explicite traditionnelle, mais, elle a redéfini et régulé la place de celle-ci, qui, dans ses démarches, vient derrière la grammaire implicite, pour l'élucider et la compléter. » (Uwizeye, $2011: 22$ ).

\section{Grammaire explicite}

20 La grammaire explicite, quant à elle, réfère à l'enseignement de règles grammaticales clairement énoncées en usant d'un métalangage qui lui est propre. Cet enseignement/ apprentissage peut être déductif ou inductif. En effet, selon Besse et Porquier :

Il s'agit de l'enseignement/apprentissage d'une description grammaticale de la langue-cible en s'appuyant expressément sur le modèle métalinguistique qui la construit (en utilisant en particulier sa terminologie, sous sa forme originale ou simplifiée) (...) la démarche peut être déductive (...) ou inductive (...) (1991: 80).

L'apprenant est donc amené à s'approprier un métalangage que l'enseignant a préalablement explicité ; il s'ensuit une application consciente à travers des exercices.

Cependant, enseigner explicitement un fait de grammaire peut-il contribuer à l'apprentissage ? L'enseignement de la grammaire en classe de FLE se fait-il de manière explicite ou implicite? D'après ces auteurs, l'enseignement explicite d'une régularité grammaticale favorise l'acquisition (1991: 80). Nous marquons notre accord avec ces auteurs concernant essentiellement l'enseignement du FLE. Nous pensons en effet que cela conviendrait à des collégiens et lycéens ayant déjà acquis des connaissances implicites sur la langue. 


\section{Grammaire inductive et démarche active de découverte (DAD) : la conceptualisation grammaticale}

La grammaire inductive renvoie, sur le plan pédagogique, à cette activité de l'apprenant qui consiste à observer un corpus d'exemples pour arriver à la règle : "L'enseignant va ou fait aller les apprenants " des exemples aux règles ", en s'appuyant sur leur capacité à remonter intuitivement d'exemples donnés aux régularités, organisations ou règles jusqu'alors inconnues » (Puren, $2001: 15)$.

Il s'agit donc d'un processus cognitif qui rend l'apprenant actif: "Les approches inductives nécessitent un travail réflexif de l'élève par l'observation d'un corpus menant à des hypothèses à confirmer » (Vincent, Dezutter, et Lefrançois, 2013 : 93). Une démarche, actuellement recommandée, est associée à cette procédure, à savoir la démarche active de découverte (désormais DAD). Initiée par Chartrand, la DAD consiste, comme son nom l'indique, à faire participer l'apprenant à découvrir les règles de grammaire en faisant appel à ses capacités cognitives. Ses erreurs répétitives servent de point de départ pour la découverte des régularités du système de la langue cible. Il s'agit donc d'une démarche expérimentale mettant en situation de réflexion (observation, expérimentation et raisonnement) des groupes d'apprenants. C'est ce que l'on nomme communément «conceptualisation grammaticale ", basée sur les fondements du constructivisme.

Selon Suzanne-G Chartrand (1996), la DAD comporte six étapes, présentées comme suit :

\section{Etape de mise en situation}

Appelée aussi situation problème, la mise en situation est nécessaire car elle sert à rappeler la notion et la placer ainsi dans son contexte d'étude. Une notion dont l'enseignant a détecté auparavant la difficulté et la non maitrise par ses apprenants (par exemple, confusion dans l'usage des pronoms relatifs).

\section{Etape de l'observation de la notion}

Les apprenants étant groupés, sont amenés à travailler sur un premier corpus de textes, guidés par l'enseignant. Celui-ci, afin de mettre en exergue la notion à étudier, donne des consignes, telles que : repérer, regrouper, classer et comparer. Des observations, des constats ainsi que des questions seront notées par les apprenants (Chartrand, 1996).

\section{Etape de la manipulation des énoncés et formulation d'hypothèses.}

En vue de l'appropriation de la notion grammaticale en question, les apprenants seront conduits à la manipuler à l'intérieur d'énoncés, par le biais de transformations et d'opérations syntaxiques, à savoir : déplacer, ajouter, soustraire, remplacer. A ce stade, ils seront capables de formuler des hypothèses quant au fonctionnement de la structure concernée : « (...) la forme du pronom relatif dépend de sa fonction (...) » (Chartrand, 1996).

\section{Etape de la vérification des hypothèses.}

Un second corpus étant distribué à l'ensemble de la classe, les apprenants pourront confirmer ou infirmer les hypothèses formulées précédemment et, susceptibles ainsi de devenir des règles et des lois générales. 


\section{Etape de la formulation de lois et de règles}

Chaque groupe est amené à énoncer avec ses propres mots et expressions la règle dégagée de son analyse, ce qui lui permet d'établir une comparaison entre les différentes règles des autres groupes. Il s'ensuit une discussion et tout en étant aidés par l'enseignant, les élèves formulent clairement les règles. Celles-ci seront vérifiées à travers des grammaires de référence.

\section{Phase d'exercisation}

Pour permettre aux apprenants une meilleure acquisition, les règles découvertes seront réinvesties à travers des exercices d'application. Chartrand propose à cet effet une typologie d'exercices allant des plus simples aux plus complexes: exercice de reconnaissance du phénomène grammatical, exercices de reconnaissance avec justification écrite, exercices de construction de phrases, exercices de correction de phrases, exercices de production de textes (idem).

Cette démarche étant explicitée, nous nous posons alors la question de savoir si elle est appliquée en classe de FLE. Malgré les critiques dont elle a fait l'objet (difficultés de l'appliquer au FLE, plage horaire conséquente...), nous pensons que ce type de démarche pourrait être fructueux dans l'enseignement de la grammaire, que ce soit au collège ou au lycée. Nous pouvons avancer comme avantages l'activité, l'autonomie et la créativité de l'élève, tel qu'annoncés par Chartrand :

(...) elle amène l'élève à être actif tout au long de son apprentissage (...) Elle lui fait expérimenter des procédures d'observation et de manipulation (...) favorisant ainsi son autonomie (...) Elle développe un esprit de rigueur et favorise le doute créateur (...) comme elle est menée par les élèves eux-mêmes (...) elle est nécessairement adaptée à leurs besoins et à leurs aptitudes (...) Enfin, elle favorise l'écoute des autres points de vue, la discussion développant ainsi les habiletés argumentatives des élèves.(idem)

\section{Analyse des documents officiels et des séances d'observation de classe}

\section{Le programme et les documents d'accompagnement}

Le programme requiert le recours à une méthode de type inductif, tel qu'on le trouve dans le document d'accompagnement au programme de $4{ }^{\text {ème }} \mathrm{AM}$ : "Conformément à l'esprit qui anime toutes les pratiques concernant les apprentissages linguistiques, les activités de grammaire obéissent à une démarche inductive » (MEN, 2013d : 41)

Pour la démarche, nous préconisons une démarche active de découverte. Le MEN (2011: 30-31) résume ci-dessous cette démarche dans l'étude des points de langue, notamment en grammaire, dans l'approche par compétences :

\begin{tabular}{|c|c|}
\hline $\begin{array}{l}\text { La } 1^{\text {ère }} \text { étape } \\
\text { ou phase de } \\
\text { découverte }\end{array}$ & $\begin{array}{l}\text { Elle consiste à observer le texte ou le corpus, et à vérifier rapidement la } \\
\text { compréhension du contenu, qui doit être guidée par des questions de } \\
\text { l'enseignant. La lecture des textes doit être faite par l'enseignant (e) ou par } \\
\text { un bon lecteur. }\end{array}$ \\
\hline
\end{tabular}




\begin{tabular}{|c|c|}
\hline $\begin{array}{l}\text { La } 2^{\text {ème }} \text { étape } \\
\text { ou phase d'analyse }\end{array}$ & $\begin{array}{l}\text { Elle concerne le repérage du fait de langue, à partir de questions posées } \\
\text { par l'enseignant (e). Il est important de laisser aux élèves le temps de la } \\
\text { réflexion. Elle porte sur l'émission d'hypothèses par les élèves. Ils doivent } \\
\text { ici réfléchir sur le fonctionnement du point de langue découvert et vont } \\
\text { émettre des hypothèses de règles de fonctionnement avec leurs propres } \\
\text { termes. }\end{array}$ \\
\hline $\begin{array}{l}\text { La } 3^{\text {ème }} \text { étape } \\
\text { Ou phase } \\
\text { d'élaboration et de } \\
\text { reformulation }\end{array}$ & $\begin{array}{l}\text { Toutes les hypothèses vraisemblables seront acceptées et vérifiées. Des } \\
\text { exemples hors du corpus pourront parfois être utiles, pour parvenir à } \\
\text { conceptualiser le point linguistique, faire une synthèse et reformuler ce à } \\
\text { quoi les élèves sont parvenus. }\end{array}$ \\
\hline $\begin{array}{l}\text { La } 4^{\text {ème }} \text { étape } \\
\text { Ou phase } \\
\text { d'appropriation }\end{array}$ & $\begin{array}{l}\text { Elle permet, par le réemploi, de vérifier la maîtrise du fait de langue, } \\
\text { sachant qu'il est nécessaire à ce stade que le réemploi se fasse en situation. }\end{array}$ \\
\hline
\end{tabular}

31 Cette démarche est assez explicitée dans le guide du professeur de $3^{\text {ème }}$ AM. En effet : «Le cheminement d'apprentissage comprend: un moment d'observation méthodique (analyse instrumentée), un moment de découverte, un moment de reformulation personnelle et un ou des moments d'évaluation " (MEN, op.cit., 2013 e : 10). Nous reconnaissons ainsi les caractéristiques de la méthode inductive où l'apprenant est amené à une démarche de conceptualisation grammaticale en vue de découvrir le fonctionnement de la langue.

La méthode inductive est donc préconisée par les programmes du moyen vu qu'ils s'inscrivent dans une optique constructiviste et socioconstructiviste ; des approches qui tendent à encourager l'activité et la réflexion de l'apprenant.

\section{Les manuels scolaires}

Le manuel -tous niveaux confondus- adopte une DAD qui favorise l'activité de l'apprenant. Ainsi, la leçon est scindée en 4 parties : j'observe, j'analyse, je retiens, je m'entraine (2015/2016: 116). A travers la phase d'observation et d'analyse du texte support, l'apprenant est amené à découvrir la notion grammaticale en question et ceci guidé par l'enseignant à travers des questions-réponses. Il est à préciser que tous les manuels suivent la même démarche. Cependant, nous pensons qu'il ne s'agit pas réellement d'une démarche active de découverte, telle qu'elle est pensée et conçue par ses concepteurs. En effet, les quelques questions présentées n'amènent pas l'apprenant à découvrir, via un véritable travail de réflexion, le fonctionnement du point de langue. Ainsi, dans le manuel de $2^{\text {ème }} \mathrm{AM}$ (2015/2016: 80), à travers la phase d'analyse de la leçon d'orthographe portant sur la formation des adverbes, nous y décelons uniquement deux questions: quel est le point commun des mots soulignés? Qu'expriment-ils? Il en est de même dans la majorité des leçons; prenons, à titre d'exemple, la leçon de vocabulaire portant sur la périphrase (idem : 88), où on trouve les questions suivantes : à qui renvoie le groupe de mots en rouge ? Qui désigne-t-il ? De telles interrogations ne favorisent guère une démarche active.

Il adopte une démarche inductive dans la mesure où l'on commence par un textecorpus et l'on termine par la règle. L'apprenant doit découvrir la règle après un travail 
de réflexion. Cette dernière est présentée à la fin de chaque leçon. Dès lors, l'apprenant ne développera point son sens de réflexion et d'analyse.

\section{Les séances d'observation}

En classe de FLE, nous remarquons d'emblée que les enseignantes des trois niveaux recourent à un enseignement explicite en ce qu'elles usent du métalangage grammatical, tel que le montre les deux extraits ci-dessous :

\section{Extrait (1)}

[...]

En: >donc cette proposition on est dans le cadre de (la proposition subordonnée<relative) $\mathrm{x} 2$

En: d'accord, ce pronom, on l'appelle le pronom relatif, le que il s'appelle le pronom (:)

En : <ce nom qui précède ou bien qui vient avant, juste avant la proposition subordonnée relative, on l'appelle l'anté cédent, il antécède, il vient avant la pièce, d'accord, on l'appelle comment ? Il s'appelle l'anté ( :) cé ( :) dent>

[...]

$[\ldots]$

En : parmi les qualifiants il y a la subordonnée relative, et pour former, obtenir, reconnaitre une subordonnée relative, déjà pourquoi on l'appelle une subordonnée relative?

E8 : madame, car elle commence par un pronom relatif $=$ En : elle commence par un pronom relatif, quels sont les pronoms relatifs que nous avons vus?

E3 : que =En : il y a « que »

$[\ldots]$

En : pronom on sait pourquoi, mais pourquoi relatif? dans le mot relatif j'entends quel verbe?

Ex $:$ relier = En : relier, qu'est c- que ça veut dire relier ?

$[\ldots]$

En: une phrase complexe, on relie 2 propositions indépendantes pour former une phrase complexe, qui dit phrase complexe dit quoi, comment $\mathrm{j}$ - reconnais une phrase complexe?

E6 $: 2$ verbes $=$ En $:$ elle a 2 verbes, si elle a 2 verbes

E6: madame, 2 propositions =En: donc elle a 2 propo sitions, l'une s'appelle comment?

E2 : principale $=\mathrm{En}:$ une proposition principale et l'autre?

Ex : subordonnée $=\mathrm{En}$ : subordonnée, c'est bon jusqu'à présent ?

[...]

D'autre part, elles suivent une démarche inductive car c'est à partir de l'observation du corpus que l'apprenant est amené à découvrir la règle, comme le montrent ces quelques extraits :

$37 \quad$ Extrait (3) ${ }^{3}$

En : (elle écrit la phrase au tableau) qui peut lire la phrase?

$[\ldots]$

E1 : <ils ont vendu la maison que leur a laissée leur grand-père.>

En : 'très bien, <donc ils ont vendu la maison que leur a laissée leur grand-père> . Vous vous rappelez bien qu'on a étudié la phrase simple et de la phrase? (quelques élèves en même temps : complexe AH). Belaid ferme ton cahier. Est-ce que c'est une phrase simple ou c'est une phrase complexe?

$[\ldots]$

E1 E2 : c'est une phrase complexe 
[...]

En : bien (.) (..) (...) elle est où la proposition principale dans cette phrase ?

$[\ldots]$

En : proposition principale, très bien =donc on revient à la phrase (5.2) donc ils ont vendu la maison (:) que leur a laissée leur ( :)?

$[\ldots]$

En : D'accord, donc maintenant <ils ont vendu ( :)>

Ex: quoi?

$[\ldots]$

$\mathrm{E}:<$ la maison du grand- père>

$[\ldots]$

En : très bien, quand j'ai posé la question j'ai dit, ils ont vendu ( :)

[...]

En : quoi ? <quand on pose la question quoi, qu'est ce qu'on trouves ?

[...]

E : COD, COD AH

En : COD, Complément d'objet $(:)=$ Ex $:$ direct.

En : bien, donc ce complément d'objet direct a été <remplacé par une proposition subor $>=\mathrm{Ex}$ : donnée AH

En : subordonnée, d'accord? Nous avons dit que la proposition qui dépend de la proposition principale, nous l'appelons la proposition subordonnée? (ils répètent subordonnée), oui ou non?

[...] comme suit : fonction.

Extrait $(4)^{4}$

La première séance d'observation a été effectuée dans une classe de deuxième année regroupant 24 apprenants. L'intitulé du projet (3) est "La légende» et celui de la séquence (2) est «personnages de la légende ». L'activité du jour est la grammaire. Comme support, l'enseignante opte pour l'utilisation de phrases. La séance se déroule

L'enseignante écrit la première phrase au tableau et demande à un élève de la lire.

-Ensuite elle fait un petit rappel sur la phrase simple et la phrase complexe (mise en situation) pour amener les apprenants à transformer la phrase support, étant une phrase complexe, en deux phrases simples, identifier la proposition principale et la proposition subordonnée relative, reconnaitre le rôle du pronom relatif (que) et sa

L'enseignante écrit la deuxième phrase support au tableau et procède de la même manière. L'objectif étant d'identifier la subordonnée relative (introduite par le relatif qui) et la fonction du pronom relatif.

Elle continue ainsi avec la $3^{\text {ème }}$ et $4^{\text {ème }}$ phrases, dont les propositions relatives sont respectivement introduites par les pronoms relatifs «où » et «dont» (étape de la manipulation d'énoncés+ formulation de lois).

A la fin de la séance, elle sollicite des phrases contenant des pronoms relatifs (qui, que, où et dont) et termine par des exercices et une intégration.

Nous pouvons conclure que la démarche de cette enseignante s'inscrit dans une DAD en ce qu'elle incite l'apprenant à découvrir lui-même, à travers un jeu de questions réponses, le fonctionnement du point de langue étudié. Toutefois, l'enseignante n'accorde pas à l'apprenant toute son autonomie réflexive dans l'étape de formulation d'hypothèses, notamment, puisqu'elle formule elle-même la règle. 
[...]

En : qui va devenir une phrase complexe (ils répètent en même temps complexe) Comment on va faire, donc puisque celui-là c'est un COD on va le remplacer par le que ici juste après le nom euh qui est répété.

[...]

En: (...) Donc c'est un COI donc quand le mot placé est un complément d'objet ( : $)=\mathrm{Ex}$ : indirect = En : on le remplace par le pronom dont, il s'écrit comment?

$[\ldots]$

[...]

En : (5.2) lis (après avoir distribué le texte support)

$[\ldots]$

En : oui on a un portrait, qui est décrit ? [...]

$\mathrm{E}:$ une jeune fille $=\mathrm{En}:$ une jeune fille, réponse complète, qui est décrit ?

$[\ldots]$

$\mathrm{E}$ : c'est une jeune fille qui est décrit $=\mathrm{En}$ : qui est décrite, très bien (5.2) oui on a décrit une jeune fille, OK ? L'essentiel une réponse complète (.)(..)(...) donc vous avez des noms que j'ai soulignés [alors je $(\mathrm{x})$ les noms si après?

[...]

En : lueur, une brillance $\mathrm{OK}$ ? (x) (.)(..)(..) alors (x) le texte (x) le portrait, vous vous souvenez qu'on $\mathrm{a}(\mathrm{x})$ le portrait et on a utilisé des $(\mathrm{x})$ ou bien des notions pour décrire, quelles sont les notions qu'on a déjà vues?

[...]

$\mathrm{E}:$ le complément du nom (si) =En : on a vu le complément du nom, très bien, on a vu aussi les participes passés, $\mathrm{OK}$ ? Et ici, fille $(\mathrm{x})$ relevez moi ce qui qualifie fille.

[...]

Ex : adjectif $=E n$ : très bien, quoi d'autre ?(x2) (x) OK ? Est-ce qu'il a pris une gentille fille ou bien une fille gentille [qui est gentille [qui ça, «qui est gentille », tu n- t'es pas par hasard trompé ? [...]

$\mathrm{E}$ : dont je t'ai parlé =En : très bien, « dont je t'ai parlé », voilà tout ça plus « dont je t'ai parlé » en plus pour « qui » toujours ah $(\mathrm{x})$

$\mathrm{E}:$ que tout le monde adore $=\mathrm{En}$ : très bien, « que tout le monde adore $~ \mathrm{j}$ - continue sur la même ligne «que tout le monde adore » on est d'accord ? =Ex : oui

$[\ldots]$

$\mathrm{E}:$ que j'aime regarder $=\mathrm{En}$ : répète $?=\mathrm{E}:$ que j'aime regarder.

[...]

En : c'est la dernière fois $(\mathrm{x})$ on peut décrire avec une proposition, on peut utiliser la proposition pour qualifier, OK? Pour qualifier les noms (5.2) regardez la proposition (x) «qui est gentille », ça commence par quel mot?(.)(..)(...) par quel mot elle commence?

$\mathrm{E}:$ qui =En : qui, très bien (.)(..)(...) comment on appelle qui ? (5.2) c'est quoi « qui »?

$[\ldots]$

E : pronom relatif $?=$ En : merci (.)(..)(..) c'est un pronom relatif (.)(..)(..)

$[\ldots]$

En : très bien, pronom relatif, OK ? Donc toutes ces propositions commencent par des pronoms relatifs, alors (x) on l'appelle comment ? Proposition?

Ex : relative $=[\ldots .$.

En : voilà, dans le conte $(\mathrm{x})().().(.$.$) (x) proposition subordonnée relative, on peut$ l'appeler? (.)(..)(..) la ? La relative, OK ? On peut l'appeler la PSR [c'est bon? Pourquoi on étudie la proposition relative Hanane $(\mathrm{x})$

$\mathrm{E}:$ pour décrire $=\mathrm{En}:$ pour décrire, pour qualifier, oui (.)(..)(...) alors donnez-moi des exemples (.)(..)(..) donnez-moi des exemples (.)(..)(...) ah, j'ai oublié une chose, comment appelle-t-on le nom décrit ? (.)(..)(..) là non (si), le nom qui est décrit?

$\mathrm{E}:$ le qualifiant $=\mathrm{En}$ : c'est pas le qualifiant, le nom qui est qualifié, on l'appelle, nom?

[...] 
En : un antécédent il peut avoir plusieurs relatives, OK ? Pour le qualifier, ici on a (x) cheveux (x), un exemple (.)(..)(...) avec les pronoms que tu veux (5.2)[ (x) allez[ $(\mathrm{x})$ non pas seulement « que » $(\mathrm{x})$ les plus faciles.

[...]

E11 : madame, la dame qui a des yeux bleus est ma maîtresse =En : la dame qui $\mathrm{a}=\mathrm{E} 11$ : des yeux bleus $=\mathrm{En}$ : est la maîtresse, très bien [(rires) $(\mathrm{x})$ sans faire de bruit, voilà, donnez moi le titre, Yahiaoui $\mathrm{AH}$.

[...]

En : Yahiaoui [(x) la relative $(5.2)$

$[\ldots]$

En : qui peut me donner la définition de la relative ?(.)(..)(...) c'est quoi la relative ? à quoi elle sert?

$[\ldots]$

\&E4: $(\mathrm{x})=\mathrm{En}$ : on est d'accord sur ça? «La relative est une proposition qui commence par un pronom relatif (.)(..)(...) c'est bon? (x) et vous avez ces dix exemples on n'a pas pris tous les pronoms relatifs, on a pris $(\mathrm{x})$ après elle sert à quoi?

En : elle sert à décrire, à qualifier à rajouter d'autres informations pour? Pour la phrase en général, $\mathrm{OK}$ ?

[...]

\section{Description et commentaire}

En classe de $3^{\text {ème }} \mathrm{AM}$, l'enseignante fait appel également à la conceptualisation des apprenants pour les amener à comprendre ce qu'est une subordonnée relative et sa fonction en langue. Nous remarquons une certaine implication de l'apprenant qui se manifeste par le jeu de questions-réponses. Cette pratique se veut dès lors inductive. Toutefois, la DAD n'a pas été appliquée à bon escient, particulièrement dans l'étape des hypothèses à laquelle l'enseignante n'accorde pas assez d'importance.

Cette deuxième séance rassemble 33 apprenants. L'intitulé de la séquence est «le portrait et l'autoportrait». L'enseignante fait un petit rappel des notions déjà vues pour brosser un portrait ou un autoportrait, puis distribue les textes supports (mise en situation). Après identification du genre du texte qui est un portrait, l'enseignante demande aux apprenants de relever les éléments qui ont servi à décrire les noms encadrés. Elle les porte au tableau et leur demande de dire de quoi il s'agit (étape de manipulation d'énoncés). Elle sollicite des exemples avec les pronoms relatifs. Enfin, celle-ci demande aux élèves de proposer le titre de la leçon. L'enseignante demande aux apprenants la définition et l'objectif de la relative (formulation de lois). L'enseignante termine par un exercice oral et un autre écrit (phase d'exercisation).

\section{Extrait (6) ${ }^{6}$}

En : [...] bon alors, avant d'entamer c- qu'on va faire aujourd'hui on va faire un petit rappel de ce que nous avons fait hier. Hier, on a continué avec la lettre ?=E1: de Hania =En : de Hania à ? =Ex : Françoise.

En : [...] que fait Hania dans sa lettre?

$\mathrm{E}:$ madame, madame $=\mathrm{En}:$ elle décrit, elle décrit quoi $?$

Ex $:$ madame, madame $=\mathrm{En}:$ pardon $?=\mathrm{E} 1:(\mathrm{x})=\mathrm{En}:$ les côtes de Béjaia, le littoral bougiote, le littoral de Béjaia, pourquoi AH Hania décrit-elle les côtes bougiotes? Pour quelle raison?

[...]

En : pour attirer Françoise, attirer Françoise à faire quoi ?

[...]

E3 : pour venir ici pour visiter $(\mathrm{x})=\mathrm{En}$ : pour attirer Françoise et lui donner envie de venir [visiter, pour qu'elle vienne passer les =E3 : séjour $=\mathrm{En}$ : quelques jours de vacances, un séjour ici à Béjaia, et pour décrire, qu'est ce que Hania a utilisé ? 
$[\ldots]$

E7 : madame, la subordonnée relative $=\mathrm{En}:$ la subordonnée relative, la subordonnée relative et les adjectifs sont là pour?

Ex : qualifier

[...]

En: (.)(..)(...) [...] on va voir comment je vais faire pour former cette subor donnée, pour l'obtenir puisque tu seras appelé à utiliser la proposition subordonnée en tant que qualifiant, pour qualifier un nom, tu dois savoir la former déjà et tu dois savoir <quel pronom relatif je dois uti li ser> pour cela tu écris exercice 1 c'est bon, alors l'exercice te dit, tu écris la question (.)(..)(...)[...]

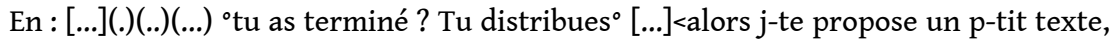
d'accord? qui est en fait un tout petit résumé de la lettre écrite par Hania à Françoise, et vous avez remarqué que dans le petit texte que je t'ai proposé tu as des propositions mises entre crochets>, >j'ai mis des propositions entre crochets<, pour l'exercice 1, concernant l'exercice 1 je te demande pour que chacune, dans chacune des propositions indépendantes mises entre crochets tu m'entoures le groupe de mots répété, tu cherches où est la répétition, tu entoures le mot ou le groupe de mots répété et tu m'indiques sa fonction dans la phrase, il est quoi dans la phrase?

[...]

En : celle mise entre crochets y a à chaque fois 2 indépendantes, donc dans les 2 indépendantes tu cherches la répé tition, [...]

En: alors, les 2 premières indépendantes lisez-les moi d'abord =Ex : madame, madame AH

$\mathrm{E}$ : Françoise a accepté l'invitation. Hania lui a envoyé cette invitation

En : où est le groupe de mots répété ? Qu'est $\mathrm{c}-$ qui est répété ?=Ex : madame, madame

E2 $:(\mathrm{x})=$ En $:$ pardon $? \& \mathrm{E} 2:$ invitation $=[\ldots]$

En: on a parlé d'un groupe de mots, c'est-à-dire plus qu'un seul, d'accord? un groupe c'est plus qu'un seul, donc ce n'est pas invitation c'est ? =Ex : cette invitation $=$ En: cette in vita tion, d'a:ccord, quelle est la fonction =Ex: madame, madame $=\mathrm{En}$ : de cette invitation dans la phrase, quelle est sa fonction, son $(\mathrm{x})$ son rôle dans la phrase[, il est quoi ?

$\mathrm{Ex}:$ madame, madame $=\mathrm{E} 5: \mathrm{COD}$

$=$ En : c'est un COD, comment je sais qu- c-t un COD $=\mathrm{Ex}$ : madame, madame $=\mathrm{En}$ : oui

E3 : je pose la question Hania a envoyé quoi?

$[\ldots .$.

=En : [...] (.)(..)(...) dans la première, les 2 premières propositions indépendantes, le mot répété est un $?=\mathrm{Ex}: \mathrm{COD}$

[...]

=En : lorsque le mot répété, il est complément d'objet direct, quel pronom relatif doit-on uti liser? [...]

=E : Françoise a accepté l'invitation que Hania lui a en vo yée, d'acccord, quel pronom relatif notre camarade a-t-il utilisé $?=\mathrm{Ex}$ : que

=En: il a utilisé le pronom relatif "que», pourquoi? Pourquoi a-t-il utilisé que ? =Ex : madame =En : il n'a pas pris qui, il n'a pas mis où, il n'a pas mis dont, pourquoi que ? =E2 : c-t un COD $=\mathrm{En}:$ parce que c'est un C.O.D, le pronom relatif que remplace un C.O.D, si il remplace un C.O.D donc lui-même c'est un C.O.D, le que C.O.D remplace quel nom?

$[. .]=.\mathrm{E} 3$ : cette invitation

$[\ldots]$

\section{Description et commentaire}

Cette troisième séance a été effectuée dans une classe d'une trentaine d'apprenants. La progression du programme est à la séquence (1) du projet (3) dont l'intitulé est "Argumenter pour inciter à la découverte ». 
51 L'enseignante préfère entamer la leçon de grammaire en utilisant le texte support (remanié) de la leçon précédente, à savoir la compréhension de l'écrit. Elle pose alors la question sur les éléments linguistiques utilisés pour la description. Outre le vocabulaire mélioratif et les adjectifs, les apprenants identifient la relative. L'enseignante enchaine alors les questions portant sur l'antécédent, le rôle du pronom relatif et la phrase complexe, tout en les explicitant.

52 Afin de comprendre parfaitement le point de langue abordé, l'enseignante donne un premier exercice constitué d'un texte avec répétitions. Il est question alors d'identifier, d'abord, le groupe de mots répété, ensuite d'indiquer sa fonction, et enfin d'employer le pronom relatif adéquat de sorte à éviter la répétition.

53 Nous remarquons la forte implication des apprenants. En effet, à travers un jeu de questions-réponses, l'enseignante mène ces derniers à la découverte des règles qui régissent l'emploi des différents pronoms.

L'analyse et la description de la pratique enseignante montre une certaine correspondance entre celle-ci et la démarche inductive prônée par les documents officiels.

L'institution éducative, en privilégiant un enseignement inductif, tend à favoriser l'activité de l'apprenant. La démarche inductive est avantageuse en ce qu'elle s'appuie davantage sur le processus d'apprentissage. En effet, elle incite l'apprenant, à travers un procédé de réflexion linguistique (conceptualisation), à développer son autonomie. Sur le plan intellectuel, il y est impliqué, ce qui le rend actif et responsable.

En classe de FLE, au moyen, les enseignantes favorisent pareillement l'activité intellectuelle des apprenants en les incitant à un raisonnement inductif, se basant sur l'observation, la manipulation et le questionnement ; autant d'opérations cognitives qui mènent d'un faire à un savoir :

Une approche de la grammaire à partir d'une démarche qui favorise le raisonnement par induction et des activités intellectuelles très variées: voir, comprendre, s'exercer, appliquer, produire, s'évaluer ou être évalué. On part du faire pour arriver au savoir. (Alma Rosa Aguilar et Delma Gonzalez, citées par Javier Suso Lopez : 39 ).

57 Toutefois, comme nous l'avons souligné plus haut, la pratique effective en classe diffère d'une enseignante à l'autre. En classe de $2^{\text {ème }} \mathrm{AM}$, à titre d'exemple, l'enseignante ne laisse pas une grande marge de réflexion aux apprenants vu qu'elle énonce elle-même la règle dès que ces derniers se trompent ou ne donnent pas la réponse.

En $3^{\text {ème }} \mathrm{AM}$, par contre, l'enseignante accorde à ses apprenants assez de temps pour réfléchir et formuler la règle ou la définition eux-mêmes. Il en est de même en classe de $4^{\text {ème }} \mathrm{AM}$ où l'enseignante accorde un maximum d'autonomie cognitive en vue de dégager les régularités régissant le point de langue étudié, à savoir la relative.

Nous estimons enfin que cette démarche inductive ne relève pas à proprement parler de la $\mathrm{DAD}$, telle que pensée par Chartrand, même si les enseignantes tendent à impliquer cognitivement leurs apprenants. 


\section{BIBLIOGRAPHIE}

BESSE, H. et PORQUIER, R., Grammaires et didactique des langues, Didier, CREDIF, 1991.

CHARTRAND, S., « Apprendre la grammaire par la démarche active de découverte », in Pour un nouvel enseignement de la grammaire, 1996, pp. 197-225.

Disponible sur [http://www.oasisfle.com/documents / apprendre_la_grammaire_par_la_demarche_active_de_decouverte.htm],

DUMARSAIS, C., « Enseigner la grammaire d'hier à aujourd'hui », 2010. Disponible sur [www.christiandumais.info/wp.../2008/.../Diversifier-et-améliorer-son-enseignement.pdf] FOUGEROUSE, M-C., « L'enseignement de la grammaire en classe de français langue étrangère ", Ela. Études de linguistique appliquée, $\mathrm{n}^{\circ}$ 122, 2001, pp. 165-178.

MARTINEAU, Y., « La grammaire et son enseignement : connaissances grammaticales et représentations de futurs enseignants de français langue seconde », 2007. Disponible sur [http:// www.archipel.uqam.ca/898/1/M9914.pdf]

MINISTERE DE L'EDUCATION NATIONALE, Document d'accompagnement au programme Français $2^{2 \grave{m e}}$ Année Moyenne, 2011.

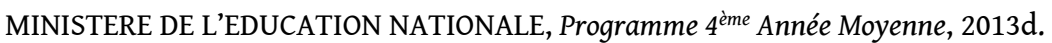

MINISTERE DE L'EDUCATION NATIONALE, Programme et document d'accompagnement Français $3^{e}$ Année Moyenne, 2012/2013b.

MINISTERE DE L'EDUCATION NATIONALE, Programme et document d'accompagnement Français $4^{e}$ Année Moyenne, 2012/2013c.

MINISTERE DE L’EDUCATION NATIONALE, Programme 4 ${ }^{\grave{m} e}$ Année Moyenne, 2013d.

MINISTERE DE L'EDUCATION NATIONALE, Manuel de français $2^{\text {ème }}$ année moyenne, ONPS, 2015/2016.

PUREN, C., « Outils et méthodologie d'analyse des manuels de langue. L'exemple des procédures d'enseignement/apprentissage de la grammaire. ». Article paru dans un cédérom de formation des enseignants des langues, réalisé dans le cadre d'un Programme de Coopération Européenne Socrates 1998-2001, « ILIAD, International Languages Inservice at a Distance ». Disponible sur [www.cilt.org.uk/iliad], 2001h.

SUSO LOPEZ, J., « La grammaire et les descriptions de la langue : la réflexion sur le fonctionnement de la langue favorise-t-elle l'apprentissage du FLE? ». Disponible sur [http:// www.ugr.es/ jsuso/publications/Chapitre\%206PhLexGrammaire.pdf]

UWIZEYE, M-L., « Les exercices de grammaire et le développement de la compétence communicative : une typologie », 2011. Disponible sur [https://core.ac.uk/download/files/ 715/30854698.pdf],.

VINCENT, F. et al., « Enseigner la grammaire selon une approche inductive ou déductive ? », 2013. Disponible sur [http://www.erudit.org/en/journals/qf/2013-n170-qf0918/70522ac/abstract/]

\section{NOTES}

1. Extrait tiré de la séance d'observation de $2^{\text {ème }}$ AM. 
2. Extrait tiré de la séance d'observation de $4^{\text {ème }}$ AM.

3. Extrait tiré de la séance d'observation de $2^{\text {ème }} \mathrm{AM}$.

4. Extrait tiré de la séance d'observation de $2^{\text {ème }}$ AM.

5. Extrait tiré d'une séance d'observation de $3^{\text {ème }} \mathrm{AM}$.

6. Extrait tiré de la séance d'observation de $4{ }^{\text {ème }} \mathrm{AM}$.

\section{RÉSUMÉS}

Nous voulons mettre en exergue la démarche inductive, préconisée par les spécialistes de l'Education en Algérie pour l'enseignement de la grammaire. Nous nous interrogeons sur le degré d'application de cette démarche en classe de FLE. Nous nous proposons d'analyser les manuels et les documents d'accompagnement de l'enseignement du français et les séances d'observation de classe pour mettre en évidence la pratique enseignante de la grammaire.

In the present study, we aim at highlighting the inductive approach advocated by specialists of education in Algeria, for the teaching of grammar. Our main inquiry is about the degree of application of this approach in FFL class. We propose to analyze the textbooks and the accompanying French teaching documents, as well as class observation sessions to highlight the teaching practice of grammar.

\section{INDEX}

Mots-clés : grammaire de FLE, démarche inductive, DADD, enseignement/apprentissage, conceptualisation grammaticale

النهج الاستقرائي, DADD, التدريس / التعلم, التصور النحويفهرس الكلمات المفتاحية:

Keywords : FFL grammar, inductive approach, DADD, teaching/learning, grammatical conceptualization

\section{AUTEUR}

\section{ZOUINA HOCINI}

Laboratoire LESMS Université de Bejaia 